\title{
Collagen type IV-related nephropathies in Portugal: pathogenic COLAA5 mutations and clinical characterization of 22 families
}

Nabais Sá M.J., Sampaio S., Oliveira A., Alves S., Moura C.P., Silva S.E., Castro R., Araújo J.A., Rodrigues M., Neves F., Seabra J., Soares C., Gaspar M.A., Tavares I., Freitas L., Sousa T.C., Henriques A.C., Costa F.T., Morgado E., Sousa F.T., Sousa J.P., da Costa A.G., Filipe R., Garrido J., Montalban J., Ponce P., Alves R., Faria B., Carvalho M.F., Pestana M., Carvalho F., Oliveira J.P. Collagen type IV-related nephropathies in Portugal: pathogenic COL4A5 mutations and clinical characterization of 22 families. Clin Genet 2015: 88: 462-467. (C) John Wiley \& Sons A/S. Published by John Wiley \& Sons Ltd, 2014

Alport syndrome (AS) is caused by pathogenic mutations in the genes encoding $\alpha 3$, $\alpha 4$ or $\alpha 5$ chains of collagen IV (COL4A3/COL4A4/COL4A5), resulting in hematuria, chronic renal failure (CRF), sensorineural hearing loss (SNHL) and ocular abnormalities. Mutations in the X-linked COLAA5 gene have been identified in $85 \%$ of the families (XLAS). In this study, 22 of 60 probands (37\%) of unrelated Portuguese families, with clinical diagnosis of AS and no evidence of autosomal inheritance, had pathogenic COLAA5 mutations detected by Sanger sequencing and/or multiplex-ligation probe amplification, of which 12 (57\%) are novel. Males had more severe and earlier renal and extrarenal complications, but microscopic hematuria was a constant finding irrespective of gender. Nonsense and splice site mutations, as well as small and large deletions, were associated with younger age of onset of SNHL in males, and with higher risk of CRF and SNHL in females Pathogenic COLAA3 or COLAA4 mutations were subsequently identified in more than half of the families without a pathogenic mutation in COLAA5. The lower than expected prevalence of XLAS in Portuguese families warrants the use of next-generation sequencing for simultaneous COLAA3/COL4A4/COL4A5 analysis, as first-tier approach to the genetic diagnosis of collagen type IV-related nephropathies.

\section{Conflict of interest}

The authors have no financial conflict of interest to disclose that could have been construed to influence the results or the interpretation of their manuscript.

\author{
M.J. Nabais Sáa,b, \\ S. Sampaio ${ }^{\mathrm{b}, \mathrm{c}}$, A. Oliveira ${ }^{\mathrm{c}}$, \\ S. Alves ${ }^{a}$, C.P. Moura ${ }^{\mathrm{d}, \mathrm{e}}$, \\ S.E. Silva ${ }^{f}$, R. Castro ${ }^{g}$, \\ J.A. Araújoh , M. Rodriguesi, \\ F. Neves', J. Seabrak, \\ C. Soares', M.A. Gasparm, \\ I. Tavares ${ }^{b, n}$, L. Freitas ${ }^{\circ}$, \\ T.C. Sousa ${ }^{p, q}$, A.C. Henriques ${ }^{r}$, \\ F.T. Costa ${ }^{\text {, }}$ E. Morgadot, \\ F.T. Sousa ${ }^{u}$, J.P. Sousa ${ }^{o, v}$, \\ A.G. da Costaw, R. Filipe ${ }^{\mathrm{x}}$, \\ J. Garridop, J. Montalban ${ }^{y}$, \\ P. Ponce ${ }^{z}$, R. Alves ${ }^{\text {aa }}$, B. Faria ${ }^{q}$, \\ M.F. Carvalho ${ }^{a b}$, M. Pestana ${ }^{b, c}$, \\ F. Carvalho and \\ J.P. Oliveira ${ }^{a, b, e}$
}

aDepartment of Genetics, Faculty of Medicine, bUnit of Research and Development of Nephrology (FCT-725), Faculty of Medicine, University of Porto, Porto, Portugal, 'Department of Nephrology, ${ }^{\mathrm{d}}$ Department of Otolaryngology, ${ }^{e}$ Medical Genetics Outpatient Clinic, ${ }^{\mathrm{f}}$ Department of Ophthalmology, Hospital de São João, Porto, Portugal, gDepartment of Nephrology, Centro Hospitalar de Trás-os-Montes e Alto Douro, Vila Real, Portugal, hDepartment of Nephrology, Hospital dos Marmeleiros, Funchal, Portugal, 'Department of Medical Genetics, Hospital Dona Estefânia, Lisboa, Portugal, 'Dialysis Clinic of Santarém, NephroCare-Portugal, Santarém, Portugal, k Department of Nephrology, Centro Hospitalar Vila Nova de Gaia/Espinho, Vila Nova de Gaia, Portugal, 'Department of Nephrology, Hospital de Braga, Braga, Portugal,

mDialysis Clinic of Restelo,

NephroCare-Portugal, Lisboa, Portugal, nDialysis Clinic of Santo Tirso, Uninefro, Santo Tirso, Portugal, ${ }^{\circ}$ Department of Nephrology, Centro Hospitalar e Universitário de Coimbra, Coimbra, 


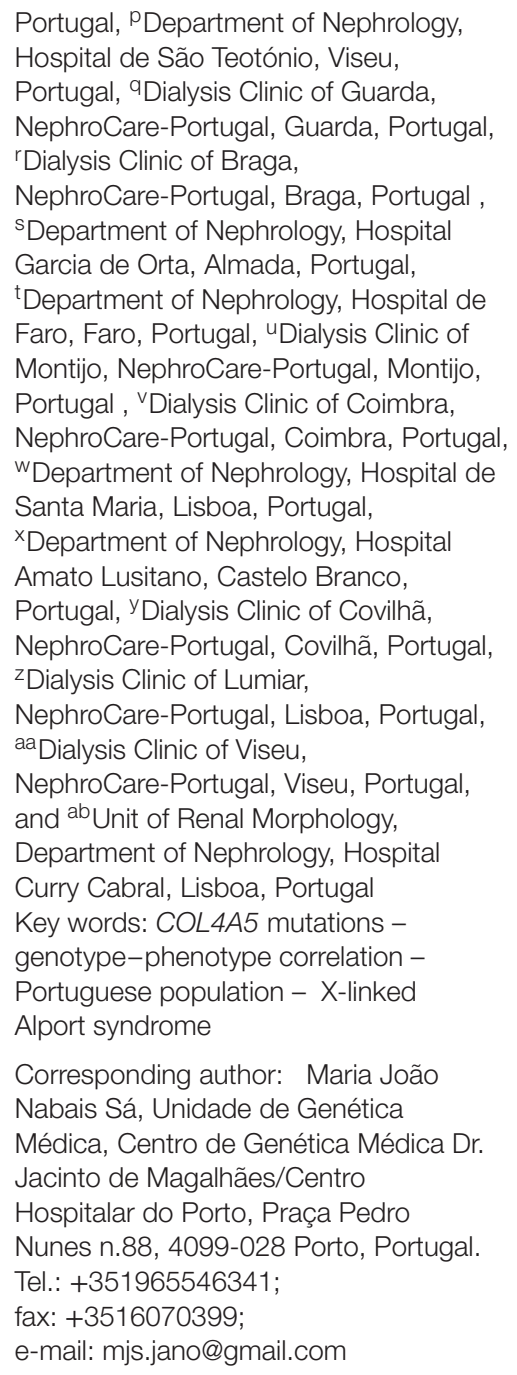

Received 16 May 2014, revised and accepted for publication 8 October 2014

Alport syndrome (AS) is caused by pathogenic mutations in the genes encoding the $\alpha 5, \alpha 4$ or $\alpha 3$ type IV collagen chains $(1,2)$, respectively, COL4A5, COLAA4 and COL4A3. The selective tissue expression of $\alpha 5 \alpha 4 \alpha 3$ (IV) heterotrimers explains the renal, cochlear and ocular involvement of AS. Although rare, AS represents an important cause of end-stage renal failure (ESRF), particularly among young adults (1).

In patients with hematuria of uncertain etiology, the clinical diagnosis of AS can be established whenever at least three of the following criteria are fulfilled (3): family history of macro/microscopic hematuria and/or chronic renal failure (CRF); typical glomerular basement membrane (GBM) lesions on electron microscopy examination; high tone sensorineural hearing loss (SNHL); characteristic ocular abnormalities (anterior lenticonus and/or white macular flecks). Immunohistochemical analysis of expression $\alpha 5$ (IV) in the epidermal basement membrane and/or of $\alpha 5 \alpha 4 \alpha 3$ (IV) in the GBM are additional AS diagnostic tools $(2,3)$. However, genetic testing has been accepted as the gold standard for the diagnosis of AS and demonstration of its mode of inheritance (2).

About $85 \%$ of AS families (1) carry pathogenic COL4A5 mutations, causing the $\mathrm{X}$-linked form of the disease (XLAS; MIM\#301050). Moreover, AS with diffuse leiomyomatosis (ATS-DL; MIM\#308940) and AS with mental retardation, midface hypoplasia and elliptocytosis (AMME; MIM\#300194) are two rare syndromes caused by microdeletions involving COL4A5 (1). The remainder of cases, which are due to pathogenic COLAA3 and/or COL4A4 mutations, are inherited as autosomal recessive (ARAS; MIM\#2013780) or dominant (ADAS; MIM\#104200) disorders. Heterozygous COLAA3 or COLAA4 mutations are identified in $40-50 \%$ of patients with thin basement membrane nephropathy (TBMN; MIM\#141200). 


\section{Nabais Sá et al.}

More than 700 disease-causing COLAA5 mutations, most of them unique to single families, have already been described (4). Whereas in hemizygous males, complex rearrangements, large and small deletions, nonsense and splice site mutations are associated with more severe phenotype and worse prognosis (5-7), no significant genotype-phenotype correlations could be identified in heterozygous females (8).

The data available on the molecular pathology of AS in Portugal were from a small number of families enrolled on a European collaborative study $(6,8)$. Our major goals are, therefore, to describe the genetic epidemiology and genotype-phenotype correlations in a large nationwide cohort of Portuguese families diagnosed with AS.

\section{Patients and methods}

Between 1 January 2009 and 30 June 2012, 60 unrelated patients with at least one of the diagnostic criterion of AS (3), and their affected or at-risk relatives who accepted genetic evaluation, were enrolled in this study for COLAA5 mutational analysis. The research protocol was approved by the Health Ethics Commission of São João Hospital Centre (Porto; Portugal).

The relevant clinical data were collected at enrolment by the referring physician, using a standardized questionnaire. Additional data were retrospectively collected by review of archive medical records, or interviewing patient's relatives. Respectively 5 (8.3\%), 17 (28.3\%), $26(43.3 \%)$ and $12(20 \%)$ of the probands fulfilled four, three, two and one diagnostic criteria, in addition to hematuria.

Genomic DNA was extracted from whole blood samples. All 53 exons of COL4A5 and corresponding exon/intron boundaries were amplified by polymerase chain reaction (PCR), using previously described primers and conditions (9). The PCR products were analyzed by Sanger DNA sequencing, using routine laboratory protocols and standard capillary electrophoresis. In addition, a commercial multiplex ligation-dependent probe amplification (MLPA) assay was used to scan for large deletions/duplications involving COLAA5 and the $5^{\prime}$ region of COL4A6. Novel variants were considered pathogenic on the basis of molecular, epidemiological, family segregation and/or bioinformatic criteria, as summarized in Table S1, Supporting Information. Whenever a novel COLAA5 variant of uncertain pathogenicity was identified, or a pathogenic COLAA5 mutation could not be identified in the proband, COLAA3 and COLAA4 were subsequently scanned for pathogenic mutations (20).

Parental genotyping was carried out, whenever possible, to confirm whether the COLAA5 mutation identified in a proband was inherited or de novo. In cases where the same novel mutation was identified in apparently unrelated probands, microsatellite polymorphic markers flanking the COLAA5-COLAA6 genes were used for haplotype analysis, as previously described $(10,11)$.

For genotype-phenotype correlation analyses, COL4A5 mutations were grouped according to the expected severity of the corresponding phenotype (7). Outcomes were compared between genders and, within each gender, between patients with large rearrangements, frameshifting, nonsense or splice donor mutations ('severe' mutations), and those with other types of mutations ('severe/moderate' mutations). To minimize ascertainment bias, the clinical phenotypes per gender and the genotype-phenotype correlations were analyzed in 'phenotyping cohorts', comprising the probands and their affected relatives subsequently enrolled in this study.

Parametric and non-parametric statistics were used, as appropriate, for comparisons of demographic variables and clinical outcomes. The data were analyzed with the IBM SPSS Statistics software version 21 (IBM Corporation; Armonk, NY).Results

Twenty-two (37\%) of the 60 probands had a pathogenic mutation in COL4A5, of which $12(57 \%)$ were novel and $9(43 \%)$ had been previously described (Table 1) $(5,9$, 12-17). Eighty percent of the probands who presented with all four diagnostic criteria, but only 1 of 3 of those who presented with two or three criteria, had a pathogenic mutation in COL4A5. Missense substitutions $(7 / 21 ; 33 \%)$, all involving glycine residues, were the most common type of mutation. The novel c. $4342 \mathrm{G}>\mathrm{C}$ (p.Gly1448Arg) missense mutation was identified in two apparently unrelated probands; in the two families, living in neighboring towns, the pathogenic mutation segregated with the same microsatellite haplotype. In 2 of $13(15 \%)$ genetic probands, the COLAA5 mutation was proven to be de novo by parental genotyping.

The identification of a pathogenic COLAA5 mutation allowed confirmation of XLAS in 43 more patients from the 22 families, increasing the size of the phenotyping cohorts to 30 males (46\%; mean age $36 \pm 16.8$ years) and 35 females (54\%; mean age $42 \pm 14.8$ years). Those two cohorts are described and compared in Table 1. Only two-thirds of the patients had undergone audiological assessment and less than $60 \%$ had been referred for specialized ophthalmological examination. This shows that appropriate screening for the extrarenal signs of AS is frequently overlooked in clinical practice (3), which might influence the diagnostic judgment. Expression of renal disease in the male cohort did not substantially differ from the usual description of XLAS in males (6); however, the prevalence of chronic kidney disease (CKD) in the female cohort was higher than previously reported (8). Microscopic hematuria was present in all cases, irrespective of gender. The risks of developing progressive CKD and ESRF were considerably higher in males $(p=0.029$ and $p<0.001$, respectively). Nevertheless, CKD was diagnosed in $62 \%$ of the heterozygous females, at young adult age in many cases. Electron micrographs of kidney biopsies, available from nine patients, showed the typical ultrastructural features of AS, irrespective of gender. Subjective hearing loss was more often reported by males ( $87 \%$ vs $46 \% ; p=0.001$ ). Anterior lenticonus and dot-and-fleck retinopathy were also more frequently diagnosed in males $(p=0.013$ and $p=0.129$, respectively). ATS-DL was diagnosed in one family, in association with a large deletion involving COLAA5, but not the COL4A6 gene (13). 
COL4A5-related nephropathies in Portugal

Table 1. Phenotypic comparisons between hemizygous males and heterozygous females for pathogenic COL4A5 mutations ( $n=65$ )

\begin{tabular}{|c|c|c|c|c|c|}
\hline & \multicolumn{2}{|c|}{ Hemizygotes $(n=30)$} & \multicolumn{2}{|c|}{ Heterozygotes $(n=35)$} & \multirow[t]{2}{*}{$p$-value } \\
\hline & & $N$ & & $N$ & \\
\hline \multicolumn{6}{|l|}{ Phenotype } \\
\hline $\begin{array}{l}\text { Age at enrolment [median (interquartile } \\
\text { range)] }\end{array}$ & $\begin{array}{c}33.5 \\
(18.5)\end{array}$ & 30 & $42(14.8)$ & 35 & 0.088 \\
\hline \multicolumn{6}{|l|}{ History of renal abnormalities } \\
\hline History of macroscopic hematuria (\%) & 61.9 & $13 / 21$ & 17.4 & $4 / 23$ & 0.002 \\
\hline $\begin{array}{l}\text { Age at diagnosis [median (interquartile } \\
\text { range)] }\end{array}$ & $5(4)$ & 11 & $3\left(11.5^{a}\right)$ & 3 & 0.501 \\
\hline History of microscopic hematuria (\%) & 100.0 & $25 / 25$ & 100.0 & $35 / 35$ & b \\
\hline Age at diagnosis [median (interquartile range)] & $\begin{array}{l}12.5 \\
(20.3)\end{array}$ & 22 & $21(26.5)$ & 28 & 0.219 \\
\hline History of proteinuria (\%) & 96.2 & $25 / 26$ & 78.8 & $26 / 33$ & 0.067 \\
\hline $\begin{array}{l}\text { Age at diagnosis [median (interquartile } \\
\text { range)] }\end{array}$ & $18(22.5)$ & 21 & $24(19)$ & 23 & 0.508 \\
\hline History of hypertension (\%) & 73.1 & $19 / 26$ & 52.9 & $18 / 34$ & 0.112 \\
\hline $\begin{array}{l}\text { Age at diagnosis [median (interquartile } \\
\text { range)] }\end{array}$ & $20(11.5)$ & 12 & 34 (22.3) & 16 & 0.002 \\
\hline History of CKD stage 2 or higher (\%) & 86.2 & $25 / 29$ & 61.8 & $21 / 34$ & 0.029 \\
\hline $\begin{array}{l}\text { Age at diagnosis [median (interquartile } \\
\text { range)] }\end{array}$ & $21(10)$ & 16 & $32(17.5)$ & 20 & 0.038 \\
\hline History of renal replacement therapy (\%) & 76.7 & $23 / 30$ & 17.1 & $6 / 35$ & $<0.001$ \\
\hline Age at onset [median (interquartile range)] & $23(19.5)$ & 21 & $40(14)$ & 6 & 0.272 \\
\hline GBM ultrastructural abnormalities & 100.0 & $6 / 6$ & 100.0 & $3 / 3$ & b \\
\hline $\begin{array}{l}\text { Age at kidney biopsy [median (interquartile } \\
\text { range)] }\end{array}$ & $20(14.5)$ & 6 & $24\left(11^{a}\right)$ & 3 & 0.933 \\
\hline \multicolumn{6}{|l|}{ Hearing loss } \\
\hline Self-noticed or subjective (\%) & 86.7 & $26 / 30$ & 45.5 & $15 / 33$ & 0.001 \\
\hline $\begin{array}{l}\text { Age at self-noticed or subjective [median } \\
\text { (interquartile range)] }\end{array}$ & $15(30)$ & 15 & $39(19)$ & 9 & 0.142 \\
\hline Audiogram (\%) & 94.1 & $16 / 17$ & 91.3 & $21 / 23$ & 0.053 \\
\hline $\begin{array}{l}\text { Age at diagnosis of hearing loss by } \\
\text { audiogram [median (interquartile range)] }\end{array}$ & $27.5(18)$ & 16 & $41(10)$ & 13 & 0.030 \\
\hline \multicolumn{6}{|l|}{ Ocular abnormalities } \\
\hline Anterior lenticonus (\%) & 33.3 & $5 / 15$ & 0.0 & $0 / 18$ & 0.013 \\
\hline $\begin{array}{l}\text { Age at diagnosis [median (interquartile } \\
\text { range)] }\end{array}$ & $19(12)$ & 5 & - & - & - \\
\hline Maculopathy (\%) & 50.0 & $10 / 20$ & 26.3 & $5 / 19$ & 0.129 \\
\hline $\begin{array}{l}\text { Age at diagnosis [median (interquartile } \\
\text { range)] }\end{array}$ & $25(19)$ & 8 & $44(22)$ & 5 & 0.180 \\
\hline Cataracts (\%) & 46.2 & $6 / 13$ & 15.0 & $3 / 20$ & 0.107 \\
\hline $\begin{array}{l}\text { Age at diagnosis [median (interquartile } \\
\text { range)] }\end{array}$ & $30(35.8)$ & 6 & $56\left(56^{a}\right)$ & 3 & 0.263 \\
\hline \multicolumn{6}{|l|}{ Other abnormalities } \\
\hline Leiomyomatosis (\%) & 3.3 & $1 / 30$ & 5.7 & $2 / 35$ & 1.000 \\
\hline $\begin{array}{l}\text { Age at diagnosis [median (interquartile } \\
\text { range)] }\end{array}$ & 24 & 1 & 18 & 1 & 0.317 \\
\hline \multicolumn{6}{|l|}{ Genotype } \\
\hline Truncating mutation (\%) & 47 & $14 / 30$ & 37.1 & $13 / 35$ & 0.437 \\
\hline
\end{tabular}

CKD, chronic kidney disease; eGFR, estimated glomerular filtration rate; GBM, glomerular basement membrane; SD, standard deviation. Age is expressed in years. eGFR is expressed in $\mathrm{ml} / \mathrm{min} / 1.73 \mathrm{~m}^{2}$.

aThe range is indicated instead of the interquartile range.

bStatistics not computed because the proportions in the comparison groups are the same.

In contrast to previous studies (5-7), we did not identify any significant differences on prevalence or severity of expression of renal disease between males carrying 'severe' or 'severe/moderate' COL4A5 mutations, although they manifested more severe extrarenal phenotypes. Conversely, the higher prevalence of
CRF and SNHL in females with 'severe' COL4A5 mutations (Table S2) was not apparent in the largest study of females with XLAS reported so far (8). This genotype-phenotype correlation might have become apparent in our cohort as result of the enrolment of females with more severe renal phenotypes. 


\section{Discussion}

This is the first study to report the clinical features, molecular pathology and genotype-phenotype correlations observed in a large number of Portuguese patients with genetically confirmed XLAS. A causative COLAA5 mutation was identified in $36.7 \%$ (22/60) of the probands. Although this mutation detection rate was significantly lower than expected, it cannot be explained by limitations of the molecular approach, as the sensitivity of Sanger sequencing combined with MLPA to identify COLAA5 mutations is higher than $90 \%(2,3)$. Because we did not exclude enrolment of probands/families without extrarenal manifestations, and the latter are less common in ADAS and absent in TBMN (18), the major explanation for these results can be the higher relative prevalence of autosomal forms of collagen type IV-related GBM nephropathies in Portuguese families with clinical diagnosis of AS (20). Our data are in agreement with those of a recent Italian study that reported the diagnosis of XLAS in only 2 out of 3 of families with AS confirmed by unbiased next generation sequencing (NGS) (19). Taken together, these observations suggest that the relative prevalence of XLAS in Southern European populations may be lower than the usual estimates in the literature (1).

Because of the heterogeneity of the collagen type IV-related GBM nephropathies, clinicians should also take a detailed three-generation family history in order to try to recognize the pattern of inheritance before initiating genetic testing for XLAS (3). Nonetheless, it should be recognized that XLAS and ARAS may be difficult to distinguish in an affected male with a small pedigree and that some hypomorphic COLAA5 mutations may manifest with microscopic hematuria, with or without ESRF, but with no SNHL or ocular involvement, thereby mimicking TBMN (1).

Identification of a pathogenic COLAA5 mutation is the most specific criterion for diagnosis of XLAS, avoiding the need for kidney and/or skin biopsies. It is pivotal for family screening and pre-symptomatic diagnosis, with obvious implications for genetic counseling; estimation of the clinical prognosis; selection of living related female donors for kidney transplantation; and prenatal and pre-implantation diagnosis. Genetic diagnosis may also permit the earlier institution of effective nephroprotective therapies, particularly by renin-angiotensin system blockade (2).

However, Sanger sequencing of all 53 COLAA5 exons and flanking intronic sequences is a laborious, time-consuming and expensive process. The recent availability of benchtop NGS platforms has offered the possibility to simultaneously analyze the COL4A5/COL4A4/COL4A3 genes at cheaper costs and with much lower turn-around times (19). This may become the first-tier genetic testing strategy to confirm the diagnosis of AS, particularly in populations where the prevalence of XLAS is not much higher than that of the autosomal forms.

\section{Supporting Information}

Additional supporting information may be found in the online version of this article at the publisher's web-site.

\section{Acknowledgements}

The authors thank the patients and their relatives for the invaluable collaboration in this study; NephroCare - Portugal, for having allowed the nationwide ascertainment of patients with Alport syndrome in their dialysis centers; and Drs Adelino Carvalho (NephroCare, Santarém), Ana Bernardo (NephroCare, Covilhã), Carlos Miguel Botelho (NephroCare, Viseu), Joana Felgueiras (NephroCare, Montijo), Liliana Pinho (Dialysis clinic, Paredes) and Luís Filipe Carvalho (NephroCare, Restelo), for their contributions to the clinical data collection of dialysis patients enrolled in this study. The authors also thank Dr Francis Earl Cook, from the Department of Epidemiology, Harvard School of Public Health, Boston, MA, for his advice and supervision of the epidemiological and statistical analyses. This work is part of the $\mathrm{PhD}$ project of Maria João Nabais Sá (supervisor: João Paulo Oliveira), to be presented at the ICBAS - 'Instituto de Ciências Biomédicas Abel Salazar' (Abel Salazar Biomedical Sciences Institute), University of Porto, Portugal. These studies were partially supported by FEDER funds through the COMPETE - 'Programa Operacional Factores de Competitividade', and by Portuguese national funds through the FCT - 'Fundação para a Ciência e a Tecnologia' (Foundation for Science and Technology) within the framework of the Cooperation Agreement between Portugal and Harvard Medical School (FCOMP-01-0124-FEDER-HMSP-ICJ/SAU-ICT/0003/2009); by a research grant from the Portuguese Society of Nephrology; by the Unit of Research \& Development in Nephrology (FCT-725) at the Department of Nephrology of the Faculty of Medicine, University of Porto, Portugal; and by the São João Hospital Centre.

Parts of these data were presented as posters at the European Human Genetics Conference 2012, Nürnberg, Germany, June 23-26, 2012 (P12.007) and at the 62nd Annual Meeting of the American Society of Human Genetics, November 6-10, 2012 San Francisco, California (3122F).

\section{References}

1. Deltas C, Pierides A, Voskarides K. Molecular genetics of familial hematuric diseases. Nephrol Dial Transplant 2013: 28 (12): 2946-2960.

2. Savige J, Gregory M, Gross O, Kashtan C, Ding J, Flinter F. Expert guidelines for the management of Alport syndrome and thin basement membrane nephropathy. J Am Soc Nephrol 2013: 24 (3): 364-375.

3. Hanson H, Storey H, Pagan J, Flinter F. The value of clinical criteria in identifying patients with X-linked Alport syndrome. Clin J Am Soc Nephrol 2011: 6 (1): 198-203.

4. International Alport Mutation Consortium, Savige J, Ars E et al. DNA variant databases improve test accuracy and phenotype prediction in Alport syndrome. Pediatr Nephrol 2014: 29 (6): 971-977.

5. Bekheirnia MR, Reed B, Gregory MC et al. Genotype-phenotype correlation in X-linked Alport syndrome. J Am Soc Nephrol 2010: 21 (5): $876-883$

6. Jais JP, Knebelmann B, Giatras I et al. X-linked Alport syndrome: natural history in 195 families and genotype- phenotype correlations in males. J Am Soc Nephrol 2000: 11 (4): 649-657.

7. Gross O, Netzer KO, Lambrecht R, Seibold S, Weber M. Meta-analysis of genotype-phenotype correlation in X-linked Alport syndrome: impact on clinical counselling. Nephrol Dial Transplant 2002: 17 (7): 1218-1227.

8. Jais JP, Knebelmann B, Giatras I et al. X-linked Alport syndrome: natural history and genotype-phenotype correlations in girls and women belonging to 195 families: a "European Community Alport Syndrome Concerted Action" study. J Am Soc Nephrol 2003: 14 (10): 2603-2610.

9. Martin P, Heiskari N, Zhou J et al. High mutation detection rate in the COLAA5 collagen gene in suspected Alport syndrome using PCR and direct DNA sequencing. J Am Soc Nephrol 1998: 9 (12): 2291-2301. 


\section{COL4A5-related nephropathies in Portugal}

10. Srivastava AK, McMillan S, Jermak $C$ et al. Integrated STS/YAC physical, genetic, and transcript map of human Xq21.3 to q23/q24 (DXS1203-DXS1059). Genomics 1999: 58 (2): 188-201.

11. Tazon-Vega B, Ars E, Burset M et al. Genetic testing for X-linked Alport syndrome by direct sequencing of COLAA5 cDNA from hair root RNA samples. Am J Kidney Dis 2007: 50 (2): 257e1-257e14.

12. Plant KE, Green PM, Vetrie D, Flinter FA. Detection of mutations in COLAA5 in patients with Alport syndrome. Hum Mutat 1999: 13 (2) $124-132$.

13. Nabais Sá MJ, Fieremans N, de Brouwer AP et al. Deletion of the 5 ' exons of COLAA6 is not needed for the development of diffuse leiomyomatosis in patients with Alport syndrome. J Med Genet 2013 50 (11): 745-753.

14. Knebelmann B, Breillat C, Forestier L et al. Spectrum of mutations in the COLAA5 collagen gene in X-linked Alport syndrome. Am J Hum Genet 1996: 59 (6): $1221-1232$.

15. Wang F, Wang Y, Ding J, Yang J. Detection of mutations in the COLAA5 gene by analyzing cDNA of skin fibroblasts. Kidney Int 2005: 67 (4) $1268-1274$
16. Renieri A, Bruttini M, Galli L et al. X-linked Alport syndrome: an SSCP-based mutation survey over all 51 exons of the COL4A5 gene. Am J Hum Genet 1996: 58 (6): 1192-1204.

17. Wang F, Zhao D, Ding J et al. Skin biopsy is a practical approach for the clinical diagnosis and molecular genetic analysis of X-linked Alport's syndrome. J Mol Diagn 2012: 14 (6): 586-593.

18. Pescucci C, Mari F, Longo I et al. Autosomal-dominant Alport syndrome: natural history of a disease due to COLAA3 or COLAA4 gene. Kidney Int 2004: 65 (5): 1598-1603.

19. Fallerini $\mathrm{C}$, Dosa L, Tita $\mathrm{R}$ et al. Unbiased next generation sequencing analysis confirms the existence of autosomal dominant Alport syndrome in a relevant fraction of cases. Clin Genet 2014: 86 (3): 252-257.

20. Nabais Sá MJ, Storey H, Flinter F et al. Collagen type IV-related nephropathies in Portugal: pathogenic COL4A3 and COL4A4 mutations and clinical characterization of 25 families. Clin Genet 2014: doi: $10.1111 /$ cge. 12521 (in press). 